\title{
La implantación de Planes de lgualdad de Oportunidades entre Mujeres y Hombres en los municipios de la Región de Murcia (España)
}

\section{The implementation of Equal Opportunity Plans between Women and Men in the municipalities of the Region of Murcia (Spain)}

\author{
INMACULADA CONCEPCIÓN SÁNCHEZ RUIZ \\ Graduada en Trabajo social, Máster Oficial en Mediación y Doctora por la Universidad de Murcia. \\ inmaculadaconcepcion.sanchez@um.es. ORCID: https://orcid.org/0000-0002-2180-4409
}

\section{MANUELA AVILÉS HERNÁNDEZ}

Profesora en el Departamento de Trabajo Social y Servicios Sociales de la Universidad de Murcia. manoliaviles@um.es. ORCID: http://orcid.org/0000-0002-8673-0690

\section{JUAN JOSÉ GARCÍA ESCRIBANO}

Profesor Titular en el Departamento de Sociología de la Universidad de Murcia. escriba@um.es. ORCID: http://orcid.org/0000-0002-7216-5803

\begin{abstract}
Resumen: En el año 2007 se aprobó, a nivel nacional, la Ley Orgánica 3/2007 para la igualdad efectiva de mujeres y hombres. Ese mismo año, en la Región de Murcia, se aprobó la Ley 7/2007 para la igualdad entre mujeres y hombres y la protección contra la violencia de género. Ambas leyes establecen la obligatoriedad a las administraciones públicas de elaborar y ejecutar planes de igualdad. El objetivo del trabajo de investigación que se recoge en este artículo es identificar y analizar los planes de igualdad de oportunidades entre mujeres y hombres que se han aprobado en los Ayuntamientos de la Región de Murcia hasta el año 2017. Teniendo en cuenta el objeto de estudio, se ha recurrido a una metodología cualitativa, centrada en la revisión documental. En la investigación han participado 40 municipios de la Región de Murcia. Se ha podido trabajar con 28 planes de igualdad implantados a nivel local desde 1992 hasta 2017. Los resultados del análisis indican que no existe un desarrollo adecuado de planes de igualdad de oportunidades entre mujeres y hombres a nivel local en la Región de Murcia. Destaca el escaso progreso institucional y se aprecia una carencia respecto a medidas específicas para prevenir las desigualdades de género en todos los ámbitos de la realidad social.
\end{abstract}

Palabras clave: políticas públicas, igualdad, planes de igualdad, administraciones locales, desigualdades de género, igualdad efectiva.

\begin{abstract}
In 2007, Organic Law 3/2007 for the effective equality of women and men was approved at the national level. That same year, in the Region of Murcia, Law 7/2007 was approved for equality between women and men and protection against gender violence. Both laws establish the obligatory nature of public administrations to prepare and execute Equality Plans. The objective of this research article is to analyse the content of the Equal Opportunity Plans between women and men that have
\end{abstract}


been approved by the City Councils of the Region of Murcia until the year 2017. Talking into account the object of study, a qualitative methodology has been used, focusing on the review of documents. Forty municipalities in the Region of Murcia have participated in the research. It has been possible to work with 28 Equality Plans implemented locally from 1992 to 2017. The results of the analysis indicate that there is no adequate development of Equal Opportunity Plans among women and men at the local level in the Region of Murcia. The low institutional progress stands out and there is a lack of specific measures to prevent gender inequalities in all areas of social reality.

Key words: public policies, equality, equality plans, local administrations, gender inequalities, effective equality.

\section{PLANTEAMIENTO DE LA INVESTIGACIÓN}

La idea de igualdad de género nace como un "constructo de la cultura democrática" (Simón, 2010, p.31). A mediados de la década de los 80, esta expresión se empieza a trasladar desde el entorno norteamericano, donde se consolida antes de alcanzar a otros países de lengua inglesa y a los organismos internacionales relacionados con derechos humanos y políticas de igualdad (Durán, 2015). Sin embargo, su desarrollo comienza mucho antes, al amparo de los movimientos feministas.

La igualdad de género se ha convertido en un asunto prioritario de la agenda pública internacional. Promovida por la Organización de Naciones Unidas (ONU) como el tercer Objetivo de Desarrollo del Milenio, y ahora como el quinto Objetivo de Desarrollo Sostenible, es considerada una pieza fundamental para el progreso del desarrollo humano y la lucha contra la pobreza a nivel global.

Según la Organización de las Naciones Unidas para la Educación, la Ciencia y la Cultura (UNESCO), la igualdad de género es un elemento central de una visión de la sostenibilidad en la cual cada miembro de la sociedad respeta a los demás y desempeña un papel que le permite aprovechar su potencial al máximo (UNESCO, 2012).

La igualdad entre mujeres y hombres es un principio jurídico universal reconocido en diversos textos internacionales sobre derechos humanos. Entre ellos destaca la Convención sobre la eliminación de todas las formas de discriminación contra la mujer, aprobada por la Asamblea General de Naciones Unidas en diciembre de 1979 y ratificada por España en 1983. En este mismo ámbito internacional, procede resaltar los avances introducidos por conferencias mundiales, como la de Nairobi de 1985 y Beijing de 1995. En la Organización Internacional del Trabajo (OIT), la igualdad entre mujeres y hombres se reconoce en la puesta en marcha de acciones positivas sobre empleo y ocupación, adoptando medidas especiales para dar solución a las discriminaciones.
La igualdad es, asimismo, un principio fundamental de la Unión Europea. En los Tratados fundacionales de 1957 ya se recogía el principio de igualdad de retribución entre mujeres y hombres para un mismo trabajo. El artículo 19 del Tratado de Funcionamiento de la Unión Europea (TFUE) prevé la adopción de legislación, al objeto de luchar contra todas las formas de discriminación, también por motivos de sexo. El Tratado de Ámsterdam, que entró en vigor en 1999, señala en su artículo 3 que "la comunidad se fijará el objetivo de eliminar las desigualdades entre el hombre y la mujer y promover la igualdad" (1997, p.25). De igual modo, se han elaborado Directivas comunitarias sobre igualdad de sexos de importante calado en materia de igualdad de oportunidades y de trato. Estas han sido trasladadas al ordenamiento de los países miembros.

En España, el artículo 14 de la Constitución Española proclama el derecho a la igualdad formal y a la no discriminación por razón de sexo. El artículo 9.2 acoge otra dimensión que hace referencia a la igualdad real. Consagra la obligación de los poderes públicos de promover condiciones para que la libertad y la igualdad del individuo y de los grupos en que se integra sean reales y efectivas.

Entre la terminología opuesta a igualdad encontramos los conceptos de desigualdad y diferencia. A nivel conceptual, la diferenciación de los sexos no debe implicar desigualdad. Sin embargo, "el sexo es para las mujeres un factor de discriminación, subordinación y desvalorización" (Parga, 2004, p.37). De hecho, la importancia de la perspectiva de género habita no solo en la posibilidad de describir y entender la existencia de dos géneros, sino en hacer visible que se trata de dos construcciones opuestas y con un estatus jerarquizado (Arconada y Leal, 2011).

Como señala Pazos (2011), la persistencia de la desigualdad entre hombres y mujeres tiene mucho que ver con las políticas públicas, ya que reflejan y potencian unas determinadas estructuras sociales, así como las normas y los valores que las fundamentan. Las políticas no son neutrales, sino que 
producen y reproducen inequidades sociales. Por tanto, los asuntos que afectan a las mujeres siempre han sido tratados con una excepcionalidad, sesgando los análisis en torno a los fenómenos demográficos, el mercado laboral, la economía sumergida o la propia desigualdad social, entre otras.

Si profundizamos un poco más en la situación de España, son numerosos los fenómenos que evidencian la desigualdad entre mujeres y hombres. Algunos ejemplos son la división sexual del trabajo, la segregación horizontal y vertical, la brecha digital de género, el techo de cristal, las cifras de violencia de género, las dificultades de conciliación y corresponsabilidad, etc.

Los datos en el ámbito de la conciliación y la corresponsabilidad muestran que, en España, en el año 2019, un 26,2\% de mujeres (de 25 a 54 años) empleadas con un hijo trabajaba a tiempo parcial, frente al 5,3\% de hombres (Instituto Nacional de Estadística, Encuesta de Población Activa, 2020).

En términos económicos, los datos que ofrece Eurostat revelan que la brecha salarial entre hombres y mujeres, es decir, la diferencia de ingresos brutos por hora entre los trabajadores y las trabajadoras, fue de un $15,7 \%$ en 2018 , situando a España en la posición catorce de veintisiete países (Eurostat, 2020).

En el ámbito de violencia de género, en España fueron asesinadas 55 mujeres en el año 2019 (Ministerio de la Presidencia, Relaciones con las Cortes e Igualdad, 2019) y 41 en lo que llevamos de este año 2020 (Ministerio de la Presidencia, Relaciones con las Cortes e Igualdad, 2020).

Con el fin de reducir las desigualdades que expresan estos datos y alcanzar la igualdad real, surge en España la Ley Orgánica 3/2007, de 22 de marzo, para la igualdad efectiva de mujeres y hombres (BOE, núm.71, de 23 de marzo de 2007). Esta Ley impulsa a las administraciones públicas, tanto de las Comunidades Autónomas como de las Entidades Locales, a cooperar para integrar el derecho de igualdad entre mujeres y hombres en sus correspondientes actuaciones de planificación y competencias.

Recoge en el capítulo I, destinado a las políticas públicas para la igualdad, el artículo 17 'Plan estratégico de igualdad de oportunidades' que señala lo siguiente: "El Gobierno, en las materias que sean de la competencia del Estado, aprobará periódicamente un Plan Estratégico de Igualdad de Oportunidades, que incluirá medidas para alcanzar el objetivo de igualdad entre mujeres y hombres y eliminar la discriminación por razón de sexo" (2007, p.12615).

El artículo 64, ubicado en el capítulo III, que aborda las medidas de igualdad en el empleo para la Administración General del Estado y para los organismos públicos vinculados o dependientes de ella, señala que:
El Gobierno aprobará, al inicio de cada legislatura, un Plan para la lgualdad entre mujeres y hombres en la Administración General del Estado y en los organismos públicos vinculados o dependientes de ella. El Plan establecerá los objetivos a alcanzar en materia de promoción de la igualdad de trato y oportunidades en el empleo público, así como las estrategias o medidas a adoptar para su consecución. El Plan será objeto de negociación, y en su caso acuerdo, con la representación legal de los empleados públicos en la forma que se determine en la legislación sobre negociación colectiva en la Administración Pública y su cumplimiento será evaluado anualmente por el Consejo de Ministros. (2007, p.12622)

Los planes de igualdad suponen un marco referencial, que señala objetivos a cumplir por parte de todos los agentes implicados, y que exige la realización de un seguimiento durante su periodo de vigencia (Federación Española de Municipios y Provincias, 2006). Del mismo modo, se entiende como una herramienta fundamental en las políticas de género, cuyo propósito es abordar las dimensiones de la discriminación y desigualdad de género, tales como las estructurales, derivadas de la división sexual en espacios y trabajo, y las culturales, derivadas de la imposición de determinados patrones de interpretación de la realidad social como los roles y estereotipos. Las características generales que, según la Federación Española de Municipios y Provincias (2006), debe cumplir un Plan de Igualdad de Oportunidades son las siguientes: colectivo e integral, transversal, dinámico, sistemático y coherente, flexible, y temporal. Estos planes de igualdad deben ser diseñados y ejecutados por profesionales de la acción social con competencia en materia de igualdad. Entre ellos, destacan los Trabajadores Sociales. De hecho, la investigación desarrollada por Pastor-Seller y Sánchez-Juárez (2014) ponía de manifiesto la estrecha relación que existe entre el agente de igualdad y el trabajador social. Según estos investigadores, surge una sinergia entre ambas figuras, como resultado de la formación y la experiencia profesional compartida.

Tras la aprobación, a nivel nacional, de la Ley Orgánica 3/2007, de 22 de marzo, para la igualdad efectiva de mujeres y hombres, la Asamblea Regional de Murcia promulga la Ley 7/2007, de 4 de abril, para la igualdad entre mujeres y hombres y de protección contra la violencia de género en la Región de Murcia (BORM, núm. 91, de 21 de abril de 2008). El artículo 11, que lleva por título "Planes de igualdad de Oportunidades entre Mujeres y Hombres", impone que el Gobierno Regional aprobará cada legislatura un Plan General que recoja, de forma coordinada y global, las líneas de 
intervención y directrices que deben orientar la actividad de los poderes públicos murcianos en materia de igualdad de oportunidades entre mujeres y hombres. Asimismo, destaca que: "en la elaboración de dicho plan el Gobierno facilitará la participación del resto de administraciones públicas de la Región de Murcia y su cumplimiento será objeto de una adecuada evaluación" (p.31922). De igual modo, "los planes deberán contener cláusulas de evaluación y seguimiento sobre la ejecución de éstos y alcance de las medidas diseñadas o grado de consecución de los objetivos previstos" (ibíd.). Este mismo artículo establece que dependerá de la Administración de la Comunidad Autónoma de la Región de Murcia, a través de las diferentes Consejerías, garantizar las medidas contempladas en los planes de igualdad y que se cumplan las leyes, órdenes o programas sectoriales que puedan impulsar el principio de igualdad de mujeres y hombres.

El artículo 15, que lleva por título "Planes municipales de igualdad de oportunidades entre mujeres y hombres", señala, por su parte, que (p.31923):

1. Las administraciones locales determinarán como objetivo prioritario la elaboración y aprobación de sus respectivos planes municipales de Igualdad de Oportunidades, que tendrán un carácter integral, y fijarán de forma coordinada y global las líneas de intervención y las directrices que deben orientar la actividad de los poderes públicos locales en materia de igualdad de oportunidades y lucha contra la violencia de género.

2. Los planes contendrán cláusulas de evaluación y seguimiento sobre la ejecución de éstos, alcance de las medidas diseñadas o grado de consecución de los objetivos previstos.

En este sentido, se pueden plantear diversas preguntas, con un especial interés en lo que sucede en las administraciones locales de la Región de Murcia:

- ¿Se han puesto en marcha planes de igualdad municipales en la Región de Murcia desde el año 2007, tal y como obliga la Ley 7/2007 para la igualdad entre mujeres y hombres?

- ¿Se han implementado las medidas establecidas en la normativa del ámbito de igualdad?

- ¿Cuál es la situación actual en la Región de Murcia, tras el compromiso legal establecido en materia de igualdad con la Ley 7/2007 para la igualdad entre mujeres y hombres?
Estas cuestiones son las que han llevado a plantear un estudio de caso que, aunque centrado en la Región de Murcia, puede servir de base para el desarrollo de futuros estudios similares en otras comunidades autónomas, que permitan comprender la realidad global que presenta España en esta materia.

\section{OBJETIVO}

El principal objetivo de la investigación que se presenta en este artículo es identificar los planes de igualdad de oportunidades entre mujeres y hombres que se han implementado en la administración pública local de la Región de Murcia hasta el año 2017. Se trata de conocer los planes de igualdad, y, por ende, las medidas en materia de igualdad implementadas en cada municipio. Esto nos permitirá analizar el cumplimiento o no del artículo 15 de la Ley 7/2007 para la igualdad entre mujeres y hombres y de protección contra la violencia de género en la Región de Murcia. A su vez, nos permitirá interpretar, aunque sea de manera indirecta, la importancia que los Ayuntamientos conceden a la promoción de la igualdad entre hombres y mujeres.

\section{METODOLOGÍA}

Se ha optado por una metodología de corte cualitativo, que se ha llevado a cabo a través de una técnica indirecta como es el análisis de documentos oficiales. En este caso, esos documentos son los planes de igualdad de los Ayuntamientos de la Región de Murcia. El marco territorial de esta investigación se ha delimitado a la intervención social en el área de igualdad, realizada en la administración pública de los 45 municipios de la Región de Murcia. Con la finalidad de obtener una visión de conjunto, la muestra fijada en el análisis de contenido cualitativo está formada por todos los planes de igualdad que se han puesto en marcha en los Ayuntamientos de la Región de Murcia.

Para la recogida de información se establecieron dos fases:

1. Solicitud de los planes de igualdad municipales a los 45 Ayuntamientos de la Región de Murcia.

La información fue solicitada individualmente a cada Ayuntamiento de manera oficial. La instancia estaba dirigida a los/as alcaldes/as, como máxima autoridad política de la administración municipal, con la finalidad de que respondieran a la solicitud a través del personal político o técnico 
especializado en la materia. Las solicitudes fueron enviadas a través de la Oficina de Asistencia en Materia de Registros de la CARM (Comunidad Autónoma de la Región de Murcia), ya que este procedimiento confirma la entrada del documento en el departamento correspondiente de cada Ayuntamiento. El tiempo de recogida de la documentación fue de un año y seis meses. Las primeras solicitudes fueron enviadas en el mes de julio de 2016. Pasados dos meses, y tras no recibir todas las respuestas esperadas, se envió una segunda solicitud de información vía online mediante correo electrónico con notificación de entrega. Finalmente, se hizo una tercera petición, en este caso telefónica, con la que concluimos el proceso en enero del año 2018.

2. Recogida de los planes de igualdad municipales.

En una segunda etapa, se llevó a cabo la recogida de los planes de igualdad municipales solicitados anteriormente. Se obtuvo un alto nivel de respuesta, ya que, de la petición de información a los 45 municipios de la Región, respondieron 40. De estos, 24 tienen o han tenido planes de igualdad en algún

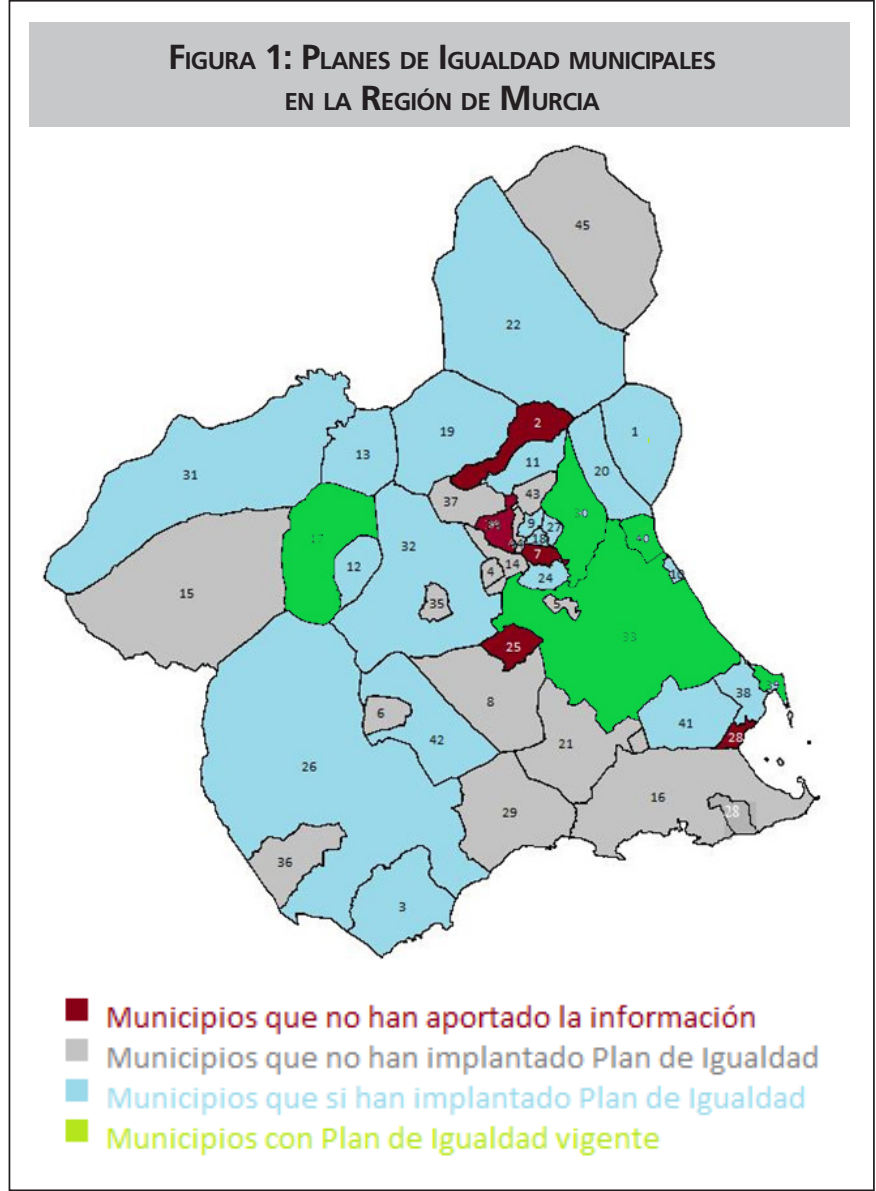

Fuente: elaboración propia momento. En total se recibieron 28 planes de igualdad, lo que quiere decir que en algunos de esos 24 municipios se han desarrollado varios planes. Los 16 Ayuntamientos restantes señalaron en su respuesta que en ningún momento habían implantado un plan de igualdad. Esto arroja ya un primer resultado, y es que, de los 40 Ayuntamientos que han respondido a nuestra solicitud, el $60 \%$ ha desarrollado planes de igualdad. El $40 \%$ restante ha incumplido la normativa vigente, al no haber puesto en marcha ninguno. En la Figura 1 se pueden observar las características de cada municipio respecto a la implantación de planes de igualdad municipales de la Región de Murcia.

\section{RESULTADOS}

En este apartado exponemos los resultados que arroja el análisis de los 28 planes de igualdad que se han obtenido en el proceso de recogida de información. Con ello, damos respuesta al objetivo del estudio que es, identificar los planes de igualdad de oportunidades entre mujeres y hombres que se han implementado en la administración pública local de la Región de Murcia hasta el año 2017.

De los 28 planes de igualdad, 6 se implementaron con anterioridad al establecimiento de la Ley 7/2007 para la Igualdad entre Mujeres y Hombres, y de Protección contra la Violencia de Género en la Región de Murcia, entre los años 1992 y 2006 . Esto quiere decir que los municipios donde se implantaron estos planes son probablemente los más orientados hacia la igualdad de género, al elaborar planes antes de la entrada en vigor de la normativa que establecía su obligatoriedad en las administraciones públicas y locales.

En lo referente al resto de planes, la mayoría se implantaron los años siguientes a la aprobación de la normativa regional, esto es, durante 2007, 2008 y, especialmente, en 2009

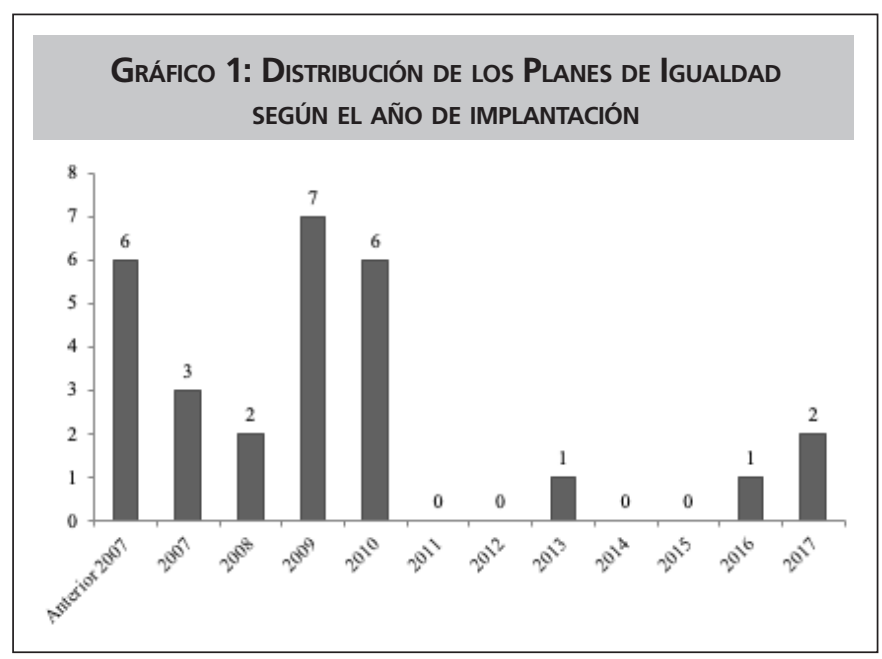

Fuente: elaboración propia 
y 2010. A partir de este último año, el interés por los planes de igualdad decrece. En el Gráfico 1 se indica, a modo de síntesis, la distribución de los 28 planes de igualdad según la fecha de su implantación hasta el año 2017.

Actualmente, hay 5 Ayuntamientos que tienen un Plan de Igualdad vigente en la Región de Murcia. Esto quiere decir que, de los 28 planes de igualdad que se han puesto en marcha, únicamente el 17,9\% están vigentes en la actualidad.

Si nos centramos en el contenido de los planes de igualdad, una unidad de análisis que se puede tomar como referencia es el área, referida a los espacios o campos de conocimiento e intervención que aparecen en el plan, y sobre los que se proyecta y desarrolla el trabajo. En el análisis de los 28 planes de igualdad se han identificado 8 áreas. Estas se explican a continuación, ordenadas en función del número de veces que aparecen en los planes.

En primer lugar, se encuentra el área de Empleo. Es considerada de gran relevancia. Hace referencia a las condiciones desiguales de acceso al empleo de mujeres y hombres, y a las iniciativas y actividades que contribuyen a disminuir este tipo de situaciones, que aún en la actualidad continúan reproduciéndose. Existen algunas diferencias respecto a cómo se denomina en cada plan. En algunos se llama Formación y Empleo, en otros Empleo y Relaciones Laborales. Uno de ellos, incluso, no habla en concreto de esta área, pero se manifiesta afín a "equilibrar el acceso y la promoción de las mujeres en el marco de una economía cambiante". Algunas de las principales medidas que contienen los planes de igualdad con respecto a esta área son:

- Sensibilizar e informar sobre igualdad en el mundo laboral.

- Incidir en la formación de las mujeres para su desarrollo personal, capacitación profesional y participación en la sociedad en condiciones de igualdad.

- Disminuir la brecha de género en el acceso a la formación y el uso de las nuevas tecnologías.

- Desarrollar actuaciones para disminuir la segregación laboral y favorecer la independencia económica de las mujeres.

La segunda área que aparece en los planes es la de Salud. La forma de designarla es variada. Encontramos unos municipios que hablan de Salud y Calidad de vida, otros municipios que la han denominado Salud y Bienestar social; también, Salud y deporte o Salud y atención social; un municipio habla de Salud laboral. A pesar de ser nombrada de diferentes formas, todas recogen actuaciones similares, tales como:
- Promocionar la salud de las mujeres en todos los ciclos de vida.

- Elaborar un estudio sobre la calidad de la salud de las mujeres, que incorpore y visibilice los riesgos en este ámbito.

- Fomentar la prevención, el diagnóstico y el tratamiento de las enfermedades de alto predominio en mujeres.

- Desarrollar actuaciones relacionadas con la salud afectiva-sexual de mujeres y hombres.

A continuación, se encuentra el área de Conciliación y Corresponsabilidad, que es denominada de la misma forma en casi todos los municipios, a pesar de que algunos añaden otras cuestiones como el reparto de responsabilidades. La conciliación es entendida como un instrumento necesario para compaginar la vida laboral, profesional y personal. La corresponsabilidad, por su parte, es señalada como la responsabilidad compartida de todas las personas, y no solamente como una imposición social al sexo femenino. Algunas de las medidas que los planes incluyen son:

- Garantizar mecanismos de conciliación de la vida personal, familiar y laboral desde la Administración Local.

- Campañas orientadas a la concienciación ciudadana sobre la importancia de lograr una conciliación laboral, familiar y personal a través de la corresponsabilidad y el reparto de tareas en el hogar.

- Charlas sobre corresponsabilidad e igualdad de oportunidades dirigidas a las asociaciones de madres y padres del municipio.

- Desarrollo de módulos sobre corresponsabilidad y reparto de tareas familiares en la escuela de madres y padres del municipio.

- Talleres educativos sobre corresponsabilidad dirigidos a los centros escolares del municipio.

El área de Violencia de género no aparece en los planes más antiguos, los que se realizaron antes de 2007, principalmente entre 1991 y 1998, pero sí en los posteriores. Refleja la prevención, sensibilización y actuación para eliminar la desigualdad social entre mujeres y hombres, y disminuir los indeseados efectos de la violencia de género, con múltiples medidas estratégicas como actividades, servicios, planes de actuación y recursos. Entre las medidas que aparecen podemos destacar:

- Realización de campañas de sensibilización y prevención de la violencia de género dirigidas a toda la ciudadanía y grupos específicos de población. 
- Garantizar e incrementar la seguridad y protección de las mujeres que se hallan en situación de violencia. Asimismo, consolidar y mejorar la atención integral a las mujeres en situación de violencia de género.

- Impulsar programas en el ámbito educativo para la prevención de la violencia de género.

- Desarrollo de talleres para el fomento de la autoestima y las habilidades sociales de las mujeres como medida de prevención de la violencia de género.

- Desarrollo de cursos de defensa personal para mujeres con el objetivo de proporcionar conocimientos y herramientas de autoprotección.

Hay dos áreas que aparecen el mismo número de veces en los planes de igualdad analizados. Se trata de Educación y de Representación, Gobernanza y Participación. Por un lado, el área de Educación está mayoritariamente unida al área de cultura y/o formación. Algunos municipios la han denominado de otras formas, si bien todos se refieren a lo mismo. La llaman Coeducación; Educación y Corresponsabilidad; Educación en valores igualitarios; Educación para la igualdad; y Educación y sensibilización. En esta área se plantean medidas en el ámbito educativo para eliminar discriminaciones y concienciar sobre la igualdad de género entre mujeres y hombres. Algunas actuaciones son:

- Realización de jornadas educativas en los distintos servicios de atención a las víctimas de violencia de género locales (juzgado, policía, guardia civil, CAVI, sanidad, etc.).

- Establecimiento de una línea de coordinación con el Centro de profesores y recursos de la Región de Murcia (CPR), para el impulso de formación del personal docente en materia de igualdad.

- Promoción para la creación de un grupo de trabajo de igualdad educativa, con los/as responsables de igualdad de los centros educativos del municipio.

- Coordinación con las personas responsables de igualdad en los diferentes consejos escolares.

El área de Representación, Gobernanza y Participación es entendida en los planes de igualdad como la participación ciudadana, y de los múltiples colectivos que hay en la comunidad. Estos están representados a todos los niveles y son partícipes de las decisiones mediante su implicación y opinión. Asimismo, los planes tienen en cuenta los presupuestos de género participativos, implantando medidas en las que se promueva la equiparación de la mujer en los puestos direc- tivos y de toma de decisiones. Los planes de igualdad muestran el gran tejido asociativo que componen los municipios de la Región de Murcia. Esta área es denominada por algunos Ayuntamientos como Asociacionismo y participación, Participación económica, Participación equilibrada en la toma de decisiones, Participación y empoderamiento. Algunas de las medidas más comunes son:

- Favorecer el acceso de mujeres y hombres a la toma de decisiones en igualdad real de condiciones.

- Incrementar la participación de las mujeres en la vida social, política, económica y cultural del municipio.

- Fomentar el asociacionismo de mujeres, promoviendo el acceso a mujeres jóvenes.

- Crear procesos de participación, espacios y canales desde los cuales las asociaciones que lo deseen puedan cooperar en la planificación, gestión y evaluación de las políticas de igualdad.

El área de Cultura y Deporte no está vinculada de la misma forma al área de deporte en todos los planes de igualdad, y tampoco al área de cultura, pero sí a otras áreas afines. Está orientada a las posibilidades que ofrece el deporte, como vehículo de formación y transmisor de valores, con el fin de superar prejuicios y estereotipos que impiden a las mujeres y a los hombres desarrollarse según sus expectativas personales y su potencial individual. Algunas de las actuaciones más destacadas son:

- Visibilizar y superar los estereotipos sexistas que conforman los roles y la imagen de las mujeres en las manifestaciones culturales, artísticas y deportivas.

- Dotar de valor el trabajo realizado tradicionalmente por las mujeres, y analizar y revisar el significado de sus aportaciones a la historia local, como elementos imprescindibles de nuestra cultura.

- Facilitar y promover la práctica deportiva de las mujeres, como instrumento que propicia la autoconciencia, la autorregulación y, en consecuencia, la autonomía y el cuidado personal.

- Apoyar y potenciar la participación de las mujeres en el deporte de competición.

El área de Visibilización y Sensibilización está menos presente en los planes de igualdad. Algunos municipios la han unido al área de violencia de género, violencia hacia las mujeres, lucha contra la violencia de género y prevención contra la violencia de género. Se entiende como el conjunto de ac- 
ciones específicas orientadas a visibilizar la situación de discriminación que sufren las mujeres en la sociedad. También a sensibilizar a toda la población con campañas específicas, orientadas a educar en igualdad y a desmontar falsos estereotipos que condicionan la vida de la mujer en sociedad. Las medidas más destacas son:

- Sensibilizar e informar a la administración local, población y medios de comunicación de la importancia de trabajar por la igualdad de oportunidades entre mujeres y hombres.

- Incorporar la perspectiva de género en todas las áreas municipales para trabajar, de manera transversal, la igualdad de oportunidades entre mujeres y hombres.

- Difundir, a través de diversos medios, los programas y servicios dirigidos a mujeres.

- Difusión del Plan de Igualdad entre la población del municipio.

A modo de resumen, se presenta el siguiente Gráfico que recoge la manera en la que las 8 áreas identificadas se distribuyen en los planes. Se indica el número de planes que aluden a cada área. Como ya se ha señalado, el área que está presente en todos los planes es la relacionada con el Empleo. La Salud aparece en 27 de los 28 planes, y la Conciliación y Corresponsabilidad está presente en 26. Esto muestra cuáles son los campos de actuación prioritarios de los planes de igualdad analizados.

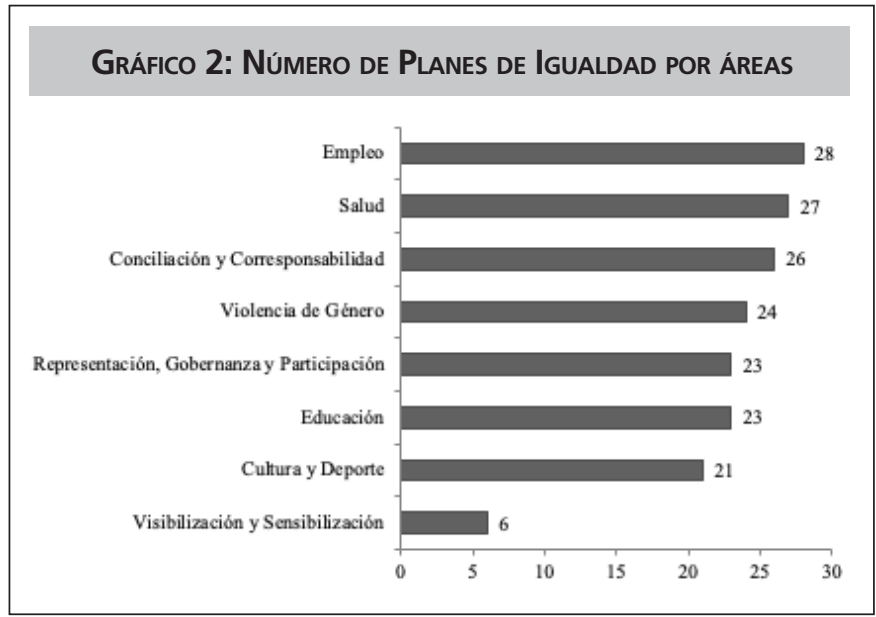

Fuente: elaboración propia

\section{CONCLUSIONES}

Al inicio de esta investigación nos planteábamos tres preguntas. En primer lugar, nos interesaba conocer si se habían puesto en marcha planes de igualdad municipales en la Región de Murcia desde el año 2007, tal y como obliga la Ley 7/2007 para la igualdad entre mujeres y hombres. Durante todo el proceso de investigación se ha trabajado con los diferentes planes. En términos generales, se ha constatado que la Región de Murcia ha tenido implementados 28 planes de igualdad distribuidos por los diferentes municipios. A pesar de que algunos de los planes sean anteriores a la aprobación de la Ley $7 / 2007$, el gran auge se presenta en el periodo que comprende desde 2008 hasta 2011. Así lo revela el hecho de que 12 planes se pusieran en marcha justo tras la entrada en vigor de la normativa, desde el año 2007 hasta el año 2010. Esto parece indicar que, de manera inmediata, la norma sí supuso un impulso en la institucionalización de las políticas de igualdad en los municipios de la Región de Murcia, tanto en la creación de estructuras como en la aprobación de planes. Este hecho contrasta con la realidad que existe actualmente, según la cual, hasta diciembre de 2018, en la Región de Murcia solo hay 5 municipios, de los 45 existentes, que disponen de un Plan de Igualdad vigente. Resulta llamativo que, en la Región de Murcia, la Ley 7/2007, en su artículo 15, señale que es una obligación de las administraciones locales, como objetivo prioritario, la elaboración de planes municipales de igualdad de oportunidades, pero no existan sanciones de tipo administrativo para aquellos municipios que no cumplen con las medidas estipuladas en la Ley. Actualmente, 40 municipios de la Región están incumpliendo el compromiso legislativo y no tienen establecido un plan de igualdad.

Con relación a la segunda pregunta, que hace referencia a la implementación de las medidas establecidas en la normativa del ámbito de igualdad, esto es, el contenido de los planes de igualdad, el análisis muestra que hay 8 áreas de actuación, aunque continúan existiendo carencias en este sentido. Al hablar de las principales medidas y/o líneas en materia de igualdad, resulta clave:

- El empleo y la formación, como actuaciones que se desarrollan para la eliminación de la brecha salarial y las desigualdades en la segregación horizontal y vertical entre mujeres y hombres.

- La conciliación y corresponsabilidad, a fin de posibilitar a mujeres y hombres la compatibilización en el ámbito familiar, laboral y escolar, sin limitar sus posibilidades y oportunidades.

- La coeducación, como fundamento del modelo educativo, que parte de los principios de igualdad entre los sexos y no discriminación.

- Las actuaciones en violencia de género, con actividades enfocadas a la necesidad de erradicar la violencia 
de género, considerada un problema a nivel mundial, y medidas necesarias para prevenir y adelantarse a cualquier situación de violencia.

Otras líneas de actuación sobre las que se está trabajando son la corresponsabilidad social, la promoción de la mujer, la visibilidad feminista, salud y calidad de vida, legislación en igualdad, desigualdades sociales, cultura y deporte. Es interesante señalar que ninguno de los 28 planes de igualdad analizados incluye el área de Emprendimiento y promoción profesional. Sin embargo, es un campo de actuación necesario en materia de igualdad, que busca potenciar la actividad empresarial de las mujeres. La finalidad es crear iniciativas relacionadas con el emprendimiento, favoreciendo el autoempleo, mediante proyectos viables.

En relación con la tercera pregunta que nos planteábamos al inicio de esta investigación, conocer la situación actual de la Región de Murcia, tras el compromiso legal establecido en materia de igualdad con la Ley 7/2007, de 4 de abril, para la igualdad entre mujeres y hombres, concluimos que esta situación resulta diversa. Es cierto que el establecimiento de dicha Ley impulsó el desarrollo institucional de las políticas de igualdad en la Región de Murcia y la elaboración de planes de igualdad como ya se ha señalado. Sin embargo, con el paso del tiempo, se han ido diluyendo las actuaciones de los gobiernos regionales y locales. Así lo revela el dato de que sólo 5 municipios tengan vigentes en la actualidad un plan de igualdad. Esto muestra que la legislación nacional y regional actual es insuficiente para conseguir la igualdad real entre mujeres y hombres, ya que marca las medidas necesarias, pero no garantiza su puesta en práctica. A pesar del desarrollo legislativo, aún son necesarios más elementos complementarios que impulsen y garanticen el desarrollo de una política de igualdad eficaz y coherente.

Es de consideración señalar que, un aspecto en las políticas de igualdad de la Región de Murcia que ha podido influir en la disminución de planes de igualdad después del año 2010 es la desaparición del Instituto de la Mujer y, con él, la casi desaparición de la figura técnica de los/as agentes de igualdad, entre cuyas funciones se encontraba la de elaborar y desarrollar planes de igualdad. Con este tipo de hechos es evidente que, desde las administraciones locales y desde el Gobierno Regional de Murcia, no se está concediendo la importancia que requiere la implementación de planes de igualdad de oportunidades entre mujeres y hombres en sus respectivos ámbitos de actuación.

Los resultados de esta investigación nos invitan a reflexionar acerca del camino que aún falta por recorrer. A pesar de que el área de igualdad se reconozca como un área de interés institucional, la resistencia al cambio es visible y los cambios estructurales son vistos como una intrusión. Para la ciudadanía, eliminar prejuicios y estereotipos patriarcales sigue siendo un reto, por lo que es complicado revertir situaciones de desigualdad que ya están normalizadas. En este sentido, determinadas instituciones patriarcales tienen peso y continúan protegiendo los criterios habitualmente presentes, si bien de manera oculta.

La carencia de medios económicos y de personal es indudablemente una traba para desarrollar las políticas de igualdad. Existe una escasez de financiación y presupuestos, situación que es agravada por los contextos de crisis. Se trata de una evidencia clara de que las prioridades políticas se orientan hacia otros sectores o ámbitos que arrojen mayor beneficio político (y quizás electoral) a corto plazo, lo que hace que no se priorice en materia de igualdad y que no se responda a las necesidades reales de esta.

Como se puede observar, alcanzar la igualdad plena entre hombres y mujeres es una tarea complicada que no tiene una respuesta concreta ni fácil pero que, poco a poco, y de manera inevitable, se abre paso a nivel institucional. La sociedad avanza y, con ella, la necesidad de implementar cambios legales y conceptuales, que se vayan ajustando a los que experimenta la sociedad.

\section{REFERENCIAS}

Arconada, M.A. y Leal González, D.A. (2011). Convivir en igualdad. Prevención de la violencia masculina hacia las mujeres en todas las etapas educativas. Madrid: UNED.

Constitución Española (1978). Boletín Oficial del Estado, núm. 311, 29 de diciembre de 1978. Recuperado de https://www.boe.es/eli/es/c/1978/12/27/(1)

Convención sobre la eliminación de todas las formas de discriminación contra la mujer. Boletín Oficial del Estado, núm. 69, 21 de marzo de 1984. Recuperado de https:// www.boe.es/buscar/doc.php?id=BOE-A-1984-6749

Decreto 314/2019 de 26 de diciembre, por el que se aprueban las normas especiales reguladoras de la concesión directa de subvenciones destinadas a los ayuntamientos de la Región de Murcia para implantar y/o actualizar planes o programas municipales de igualdad como prevención de la violencia de género. Boletín Oficial de la Región de Murcia, núm. 8, de 11 de enero de 2020. Recuperado de https://www.borm. es/services/anuncio/ano/2020/numero/160/pdf?id=782519

Decreto 4/2020, de 30 de enero, por el que se regula la Composición, Organización y Régimen de Funcionamiento del 
Observatorio de Igualdad en la Comunidad Autónoma de la Región de Murcia. Boletín Oficial de la Región de Murcia, núm. 29, de 5 de febrero de 2020. Recuperado de https://www.borm.es/services/anuncio/ano/2020/numero/563/pdf?id=782935

Durán, M.A. (2015). Introducción al Bloque IV. Relaciones de género. En C. Torres Albero (Ed.). España 2015. Situación social (pp. 405-410). Madrid: Centro de Investigaciones Sociológicas, CIS.

Eurostat (2018). Los salarios en Europa, datos y gráficos. Recuperado de https://www.epdata.es/datos/brecha-salarial-hombres-mujeres-espana-datos-graficos/320

Federación Española de Municipios y Provincias (2006). Guía para Elaborar Planes Locales de Igualdad. Recuperado de http://femp.femp.es/files/566-182-archivo/Guia\%20elaboracion \%20Planes\%20Locales\%20lgualdad.pdf

Instituto Nacional de Estadística (2020). Encuesta Población Activa. Recuperado de https://www.ine. es/ss/Satellite? L=es ES\&c=INESeccion $C \& c i d=1$ $259925472720 \& p=1254735110672$ \&pagename=ProductosYServicios\%2FPYSLayout

Ley $3 / 2007$, de 22 de marzo, para la igualdad efectiva entre mujeres y hombres. Boletín Oficial del Estado, núm. 71, de 23 de marzo de 2007. Recuperado de https://www. boe.es/buscar/pdf/2007/BOE-A-2007-6115-consolidado. pdf

Ley $7 / 2007$, de 4 de abril, para la igualdad entre mujeres y hombres y de protección contra la violencia de género en la Región de Murcia. Boletín Oficial de la Región de Murcia, núm. 91, de 21 de abril de 2008. Recuperado de https://www.boe.es/boe/dias/2008/07/22/pdfs/A3191931930.pdf

Ministerio de la Presidencia, Relaciones con las Cortes e Igualdad (2019). Mujeres víctimas mortales por violencia de género en España a manos de sus parejas o exparejas. Recuperado de https://violenciagenero.igualdad.gob.
es/violenciaEnCifras/victimasMortales/fichaMuj eres/pdf/ Vmortales 20194 12(2).pdf

Ministerio de la Presidencia, Relaciones con las Cortes e Igualdad (2020). Mujeres víctimas mortales por violencia de género en España a manos de sus parejas o exparejas. Recuperado de https://violenciagenero.igualdad.gob. es/violenciaEnCifras/victimasMortales/fichaMujeres/pdf/ Vmortales 202015 06.pdf

Parga, L. (2004). Una mirada al aula. La práctica docente de las maestras de escuela primaria. Barcelona - México D.F: Plaza y Valdés Editores. Universidad Pedagógica Nacional.

Pastor-Seller, E. y Sánchez-Juárez, M.D. (2014). Trabajo Social y Agente de Igualdad: Análisis de competencias, sinergias, potencialidades y oportunidades laborales y profesionales. Portularia, 14(1), 35-47. Recuperado de http:// rabida.uhu.es/dspace/bitstream/handle/10272/7579/Trabajo-Social-y.pdf? sequence $=2$

Pazos, M. (2011). Roles de género y políticas públicas. Sociología del trabajo, 73, 5-23. Recuperado de https://recyt. fecyt.es/index.php/sociologiatrabajo/article/view/55571

Real Decreto-Ley 9/2018, de 3 de agosto, de medidas urgentes para el desarrollo del pacto de estado contra la violencia de género. Boletín Oficial del Estado, núm. 188, de 4 de agosto de 2018. Recuperado de https://www.boe.es/ boe/dias/2018/08/04/pdfs/BOE-A-2018-11135.pdf

Simón, M.E. (2010). La igualdad también se aprende: cuestión de coeducación. Madrid: Narcea.

Tratado de Ámsterdam (1997). Boletín Oficial del Estado, núm. 109, 7 de mayo de 1999. Recuperado de https:// www.boe.es/buscar/doc.php?id=BOE-A-1999-10228

UNESCO (2012). World atlas of gender equality in education. París: Organización de las Naciones Unidas para la Educación, la Ciencia y la Cultura. Recuperado de https:// europa.eu/capacity4dev/public-gender/documents/unesco-world-atlas-gender-equality-education 